\title{
Short Helical Antenna Array Fed from a Waveguide
}

\author{
HISAMATSU NAKANO, MEMBER, IEEE, NOBUYOSHI ASAKA, AND JUNJ YAMAUCHI
}

\begin{abstract}
An array consisting of short helical antennas is fed from a single rectangular waveguide. Stubs are introduced near the array elements in order to make a matching condition between the array elements and the waveguide. On the basis of a transmission line theory, a coupling phase and a coupling factor are determined. The mechanical rotation is applied to each array element so that in-phase condition at the aperture may be formed. Excellent agreement between the calculated and experimental results is demonstrated in the arrays of five, seven, and nine helical antennas.
\end{abstract}

\section{INTRODUCTION}

$\mathbf{J}_{\mathrm{n}}$ D.' KRAUS FOUND a helical antenna operating in the axial mode in 1947 [1] and established useful expressions for the design of this antenna [2]. These are based on experimental results. Subsequently, Kornhauser tried to obtain the radiation pattern by a theoretical way and pointed out that the exact current distribution had to be determined for the calculation of the radiation pattern [3]. Ever since, considerable efforts have been made to explain the radiation characteristics of the helical antenna [4], [5], [6]

Recently the authors theoretically revealed the radiation characteristics of the helical antenna, including the current distribution, radiation pattern, power gain, input impedance, and axial ratio, by solving an integral equation [7], [8], [9]. As a result, it was found that the axial ratio shows a wavelike change as the number of helical turns is increased. In addition, it was revealed that a helical antenna with a small number of turns has good circularity, provided the antenna possesses only a decaying current distribution.

The purpose of this paper is to present an application of helical antennas with a small number of turns to an array for beam formulation. Since the helical antenna is conventionally fed from a coaxial line, its array needs coaxial lines of which the number is basically equal to that of the array elements. In addition, the same number of phase shifters and attenuators is required. To eliminate this type of complicated feeding structure, an array fed from a single waveguide has been proposed as a possible solution [10], [11]. In this paper the array of helical antennas fed from a waveguide is fully investigated. In order to facilitate easier design of the array, stubs are introduced near the array elements.

In the present design procedure, a transmission line theory is used to determine a so-called coupling phase, as in array design of slot antennas [12]. To obtain in-phase condition at the aperture, mechanical rotation of helical array elements is adopted instead of using phase shifters. Some experimental results are presented with the calculated ones.

Manuscript received July 24, 1983; revised March 12, 1984.

The authors are with the Department of Electrical Engineering, College of Engineering, Hosei University, 3-7-2 Kajino-cho, Koganei City, Tokyo, 184, Japan.

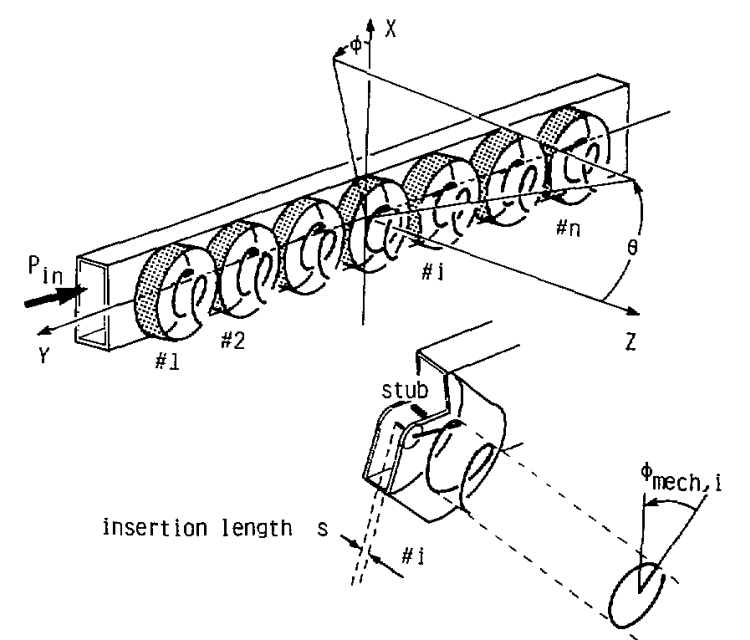

Fig. 1. Configuration of helical antenna array fed from a waveguide.

\section{CONFIGURATION}

The array of helical antennas fed from a waveguide is shown in Fig. 1. The array element has a helical section and a linear section. The number of helical turns is taken to be 1.5 turns [13], because it has been numerically shown that the helical section has a smoothly decayed current to radiate a circularly polarized wave [14]. The helical section is surrounded with a cavity in order to reduce the mutual couplings among the array elements [15], [16]. The linear section is inserted into the waveguide through a small hole and excited by $\mathrm{TE}_{10}$ mode in a rectangular waveguide. The portion of the power in the waveguide is transmitted in sequence as the radiation power from the helical section into free space, and the remaining power travels toward the end of the waveguide where it is absorbed by a dummy load. If the array element is conventionally fed from a coaxial line, the array needs coaxial lines of which the number is basically equal to that of the array elements. In addition, the same number of phase shifters and attenuators is required. The use of a waveguide eliminates this type of complicated feeding structure.

The power efficiency in the array system using the waveguide is defined as $\eta=P_{\text {rad }} / P_{\text {in }}$, where $P_{\text {rad }}$ is the total radiation power from the array elements and $P_{\text {in }}$ is the input power to the waveguide. In a case where the input power $P_{\text {in }}$ and the power efficiency $\eta$ are taken to be constant, the radiation power per array element is increased as the number of the array elements is decreased. Consequently, it becomes necessary for each array element to have a longer insertion length. This causes difficulty in designing the array, due to larger reflected waves in the waveguide. To eliminate this kind of difficulty, tuning stubs are introduced near the array elements. This leads to a smooth flow of the power in the waveguide and simplifies the array design. 


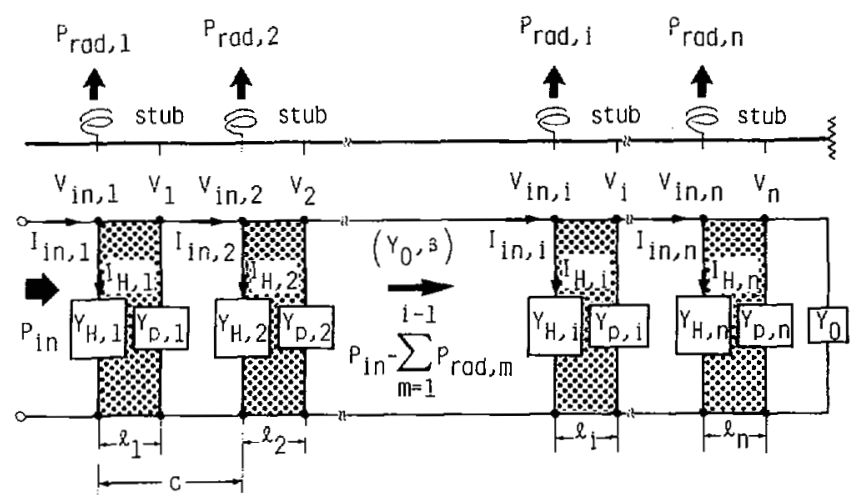

Fig. 2. Equivalent circuit of the array consisting of $n$ helical antennas.

\section{DESIGN PROCEDURE}

The design is carried out using a transmission line theory. Fig. 2 shows the equivalent circuit of the array consisting of $n$ helical antennas, where $Y_{H, i}\left(=G_{H, i}+j B_{H, i}\right)$ is the admittance of the $i$ th array element, and $Y_{p, i}\left(=j B_{p, i}\right)$ is the admittance of the $i$ th tuning stub. For convenience, a set of $Y_{H, i}$ and $Y_{p, i}$ is called an $i$ th cell. Each cell is adjusted so that the input power flows smoothly toward the end of the waveguide. In other words, the input impedance of each cell is matched to the characteristic impedance of the waveguide, $Z_{0}\left(=1 / Y_{0}\right)$. The cell in this situation is called a "matched cell."

\section{A. Coupling Phase $\phi_{i}$}

We assume that the mutual couplings among the array elements on the waveguide are negligible, and first determine a phase difference $\theta_{i}$ between the terminal voltage of the $i$ th tuning stub, $V_{i}$, and the terminal voltage of the $i$ th array element, $V_{\mathrm{in}, i}$. $V_{i}$ is given by

$$
V_{i}=\left(\cos \beta l_{i}+j Z_{0} Y_{H, i} \sin \beta l_{i}\right) V_{\mathrm{in}, i}-j Z_{0} I_{\mathrm{in}, i} \sin \beta l_{i}
$$

where $\beta=2 \pi / \lambda g$ ( $\lambda g$ is wavelength in waveguide) is the phase constant in the waveguide, and $l_{i}$ is the distance between the $i$ th array element and the $i$ th tuning stub. $I_{\mathrm{in}, i}$ in the matched cell is given by

$$
I_{\mathrm{in}, i}=\frac{V_{\mathrm{in}, i}}{Z_{\mathrm{0}}}
$$

Substituting (2) into (1), we have

$$
\begin{aligned}
\frac{V_{i}}{V_{\mathrm{in}, i}}= & \left\{\left(\cos \beta l_{i}-Z_{0} B_{H, i} \sin \beta l_{i}\right)^{2}\right. \\
& \left.+\left(Z_{0} G_{H, i}-1\right)^{2} \sin ^{2} \beta l_{i}\right\}^{1 / 2} e^{j \theta_{i}}
\end{aligned}
$$

where the phase difference $\theta_{i}$ is

$$
\theta_{i}=\tan ^{-1} \frac{\left(Z_{0} G_{H, i}-1\right) \tan \beta l_{i}}{1-Z_{0} B_{H, i} \tan \beta l_{i}}
$$

Secondly, we determine the distance between the array element and tuning stub, $l_{i}$. The matching condition of the $i$ th cell is expressed by

$$
\begin{aligned}
Y_{0}= & G_{H, i}+j B_{H, i} \\
& +Y_{0} \frac{Z_{0}\left(j B_{p, i}+Y_{0}\right)+j \tan \beta l_{i}}{1+j Z_{0}\left(j B_{p, i}+Y_{0}\right) \tan \beta l_{i}} .
\end{aligned}
$$

From the identities of the real parts and the imaginary parts in the right and left sides in (5),

$$
l_{i}=\frac{1}{\beta} \tan ^{-1} \frac{G_{H, i}}{B_{H, i}-\sqrt{\left(G_{H, i}^{2}+B_{H, i}^{2}\right)\left(1-Z_{0} G_{H, i}\right)}} .
$$

Thirdly, we determine the phase of current flowing to the $i$ th array element, $\phi_{i}$. The $\phi_{i}$ is designated as a "coupling phase."

The current flowing to the $i$ th array element, $I_{H, i}$, is given by

$$
I_{H, i}=\left(G_{H, i}+j B_{H, i}\right) V_{\mathrm{in}, i} \text {. }
$$

Equation (7) is transformed into (8) using $V_{\mathrm{in,1}}$ as a reference voltage

$$
I_{H, i}=\sqrt{\left(G_{H, i}^{2}+B_{H, i}^{2}\right)} e^{j h_{i}}\left|\frac{V_{\mathrm{in}, i}}{V_{\mathrm{in}, 1}}\right| e^{j \psi_{i}}\left|V_{\mathrm{in}, 1}\right|
$$

where

$$
h_{i}=\tan ^{-1} \frac{B_{H, i}}{G_{H, i}}
$$

and $\psi_{i}$ is a phase difference between $V_{\mathrm{in}, i}$ and $V_{\mathrm{in}, 1} . V_{\mathrm{in}, i /}$

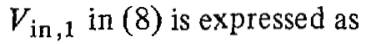

$$
\begin{aligned}
\frac{V_{\mathrm{in}, i}}{V_{\mathrm{in}, 1}}= & \left(\frac{V_{1}}{V_{\mathrm{in}, 1}} \frac{V_{\mathrm{in}, 2}}{V_{1}}\right)\left(\frac{V_{2}}{V_{\mathrm{in}, 2}} \frac{V_{\mathrm{in}, 3}}{V_{2}}\right) \cdots \\
& \cdot\left(\frac{V_{i-1}}{V_{\mathrm{in}, i-1}} \frac{V_{\mathrm{in}, i}}{V_{i-1}}\right) .
\end{aligned}
$$

We assume that the waveguide is lossless and recall that each cell is in the condition of the matched cell. Then,

$$
\begin{aligned}
\frac{V_{\mathrm{in}, i}}{V_{\mathrm{in}, 1}}= & \frac{V_{1}}{V_{\mathrm{in}, 1}} e^{-i \beta\left(d-l_{1}\right)} \frac{V_{2}}{V_{\mathrm{in}, 2}} e^{-j \beta\left(d-l_{2}\right) \ldots} \\
& \cdot \frac{V_{i-1}}{V_{\mathrm{in}, i-1}} e^{-i \beta\left(d-l_{i-1}\right)}
\end{aligned}
$$

where $d$ is the distance between the array elements. Equation (8) is expressed using (11) as follows:

$$
\begin{aligned}
I_{H, i}= & \sqrt{\left(G_{H, i}^{2}+B_{H, i}^{2}\right)}\left|\frac{V_{1}}{V_{\mathrm{in}, 1}}\right|\left|\frac{V_{2}}{V_{\mathrm{in}, 2}}\right| \ldots \\
& \cdot\left|\frac{V_{i-1}}{V_{\mathrm{in}, i-1}}\right|\left|V_{\mathrm{in}, 1}\right| e^{j \phi_{i}}
\end{aligned}
$$

where the coupling phase is

$$
\begin{aligned}
\phi_{i}= & \psi_{i}+h_{i}=\theta_{1}-\beta\left(d-l_{1}\right)+\theta_{2}-\beta\left(d-l_{2}\right) \\
& +\cdots+\theta_{i-1}-\beta\left(d-l_{i-1}\right)+h_{i} .
\end{aligned}
$$

Finally, substituting (4), (6), and (9) into (13), we have

$$
\phi_{i}=\sum_{m=1}^{i-1} \Delta_{m}-(i-1) \beta d+\tan ^{-1} \frac{B_{H, i}}{G_{H, i}}
$$

where

$$
\begin{aligned}
\Delta_{m}= & \tan ^{-1}\left[-G_{H, m}^{2} Z_{0} /\left[-B_{H, m}\left(2-G_{H, m} Z_{0}\right)\right.\right. \\
& \left.\left.+2 \sqrt{\left(\bar{G}_{H, m}^{2}+B_{H, m}^{2}\right)\left(1-G_{H, m} Z_{0}\right)}\right\}\right]
\end{aligned}
$$




\section{B. Coupling Factor $\gamma_{i}$}

The coupling factor in the $i$ th array element, $\gamma_{i}$, is defined as the ratio of $P_{\mathrm{rad}, i} / P_{\mathrm{in}, i}$, where $P_{\mathrm{rad}, i}$ is the radiation power from the $i$ th array element and $P_{\text {in }, i}$ is the input power to the $i$ th array element. In the condition of the matched cell

$$
\gamma_{i}=\frac{P_{\mathrm{rad}, i}}{P_{\mathrm{in}, i}}=\frac{G_{H, i}\left|V_{\mathrm{in}, i}\right|^{2}}{Y_{0}\left|V_{\mathrm{in}, i}\right|^{2}}=G_{H, i}^{*}
$$

where the asterisk is the notation of normalization by the characteristic admittance of the waveguide, $Y_{0}\left(=1 / Z_{0}\right)$. Since $P_{\mathrm{in}, 1}$ is equal to $P_{\text {in }}$ in the condition of the matched cell, the coupling factor in the first element $\gamma_{1}$ is expressed using the power ef ficiency $\eta$.

$$
\gamma_{1}=\frac{P_{\mathrm{rad}, 1}}{P_{\mathrm{in}, 1}}=\frac{P_{\mathrm{rad}}}{P_{\mathrm{in}}} \frac{P_{\mathrm{rad}, 1}}{P_{\mathrm{rad}}}=\eta \frac{P_{\mathrm{rad}, 1}}{\sum_{m=1}^{n} P_{\mathrm{rad}, m}} .
$$

The coupling factor in the second element is given by

$$
\gamma_{2}=\frac{P_{\mathrm{rad}, 2}}{P_{\mathrm{in}, 2}}=\frac{\gamma_{1}\left(P_{\mathrm{rad}, 2} / P_{\mathrm{rad}, 1}\right)}{1-\gamma_{1}} .
$$

Generally, the coupling factor in the $i$ th element is

$$
\gamma_{i}=\frac{\gamma_{1}\left(P_{\mathrm{rad}, i} / P_{\mathrm{rad}, 1}\right)}{1-\gamma_{1} \sum_{m=1}^{i-1}\left(P_{\mathrm{rad}, m} / P_{\mathrm{rad}, 1}\right)}=G_{H, i}^{*} .
$$

\section{Determination of Design Parameters}

The array of helical antennas is realized by using (14) and (19). Hence, fundamental information on the admittance of the helical antenna in the equivalent circuit presentation, $G_{H}^{*}+$ $j B_{H}^{*}\left(=G_{H} / Y_{0}+j B_{H} / Y_{0}\right)$, is required in advance. The admittance of the helical antenna depends on the insertion length of linear section into the waveguide as shown in Fig. 1 . The admittance is experimentally obtained by a conventional standing wave method, which is used in determination of unknown impedance (17). An example of the admittance graph will be shown in Fig. 3 . Once the relation between the admittance and the insertion length is known, the insertion length which satisfies a specified value of conductance $G *$ *an be determined, giving a value of susceptance $B_{H}^{*}$. After preparing the admittance graph, we calculate the design parameters as follows.

First, the power efficiency $\eta$ is chosen to be a specific value in (17). Secondly, we give the aperture distribution or the radiation power distribution at the aperture, $P_{\mathrm{rad}, i}(i=1,2, \cdots, n)$ in $(17)$, so as to achieve a desired radiation pattern with a specific sidelobe level. Thirdly, $\gamma_{i}(i=1,2, \cdots, n)$ is calculated using (19). $\gamma_{i}$ is equal to $G_{H, i}^{*}$. The insertion length which realizes $G_{H, i}^{*}$ is obtained from the admittance graph made in advance, giving a value of $B_{H, i}^{*}$. Therefore, from (14) the coupling phase $\phi_{i}$ can be calculated.

Finally, the mechanical rotation angle of the $i$ th array element, $\phi_{\mathrm{mech}, i}$, is given by using the relation of $\phi_{i}+\phi_{\mathrm{mech}, i}=0$. The mechanical rotation of each array element leads to the inphase condition at the aperture of the array. For example, if $\phi_{i}=-\delta$ at the $i$ th array element shown in Fig. 1, then the mechanical rotation must be made counterclockwise by $\delta$ (rad).

In practice we first insert the $n$th array element (the last array element), which satisfies $\gamma_{n}=G_{H, n}^{*}$, into the waveguide. After rotating the $n$th array element by $\phi_{\mathrm{mech}, n}$, we tune it

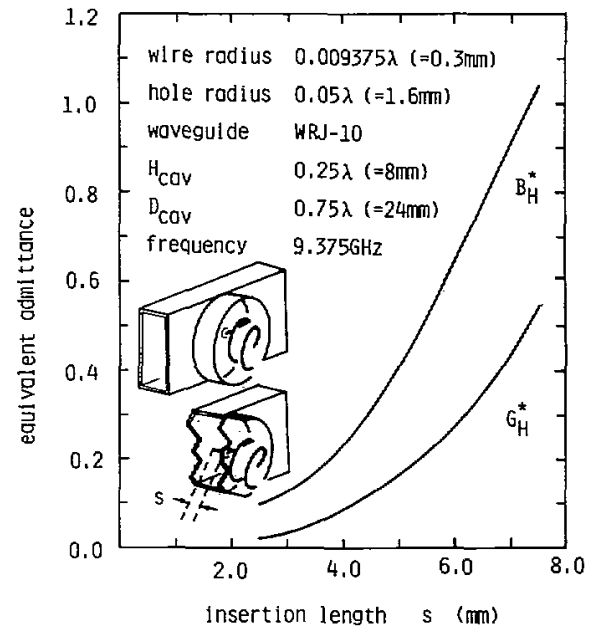

Fig. 3. Experimental result of equivalent admittance of the helical array element with a cayity, $G_{H}^{*}+j B_{H}^{*}$ versus the insertion length.

by the $n$th stub. Subsequently we insert the $(n-1)$ th array element, which satisfies $\gamma_{n-1}=G_{H, n-1}^{*}$, with rotation angle of $\phi_{\text {mech, } n-1}$ into the waveguide and tune it by the $(n-1)$ th stub. Similarly, the insertion, rotation and tuning are done one by one for the $(n-2)$ th, $(n-3)$ th, $\cdots$, and first array elements.

\section{EXPERIMENTAL RESULTS}

The operating frequency is chosen to be $9.375 \mathrm{GHz}$, which is a typical frequency in the $X$ band, and a waveguide of WRJ-10 is used to feed the array of 1.5-turn helical antennas. The parameters of the helical antenna must be chosen so that the circularly polarized beam may be obtained. The circumference of the helical cylinder is $C=1 \lambda$ ( $\lambda$ is free space wavelength) $=3.2 \mathrm{~cm}$. The pitch angle and the wire radius are $\alpha=12.5^{\circ}$ and $\rho=$ $0.009375 \lambda=0.3 \mathrm{~mm}$, respectively.

Fig. 3 shows an experiment result of equivalent admittance of the helical array element with a cavity, $G_{H}^{*}+j B_{H}^{*}$ versus the insertion length. The diameter and height of the cavity are $D_{\text {cav }}=0.75 \lambda$ and $H_{\text {cav }}=0.25 \lambda$, respectively. The axial ratio is $1.7 \mathrm{~dB}$ regardless of insertion length.

Fig. 4 shows experimental radiation patterns of the single array element with the mechanical rotation of $\phi_{\mathrm{mech}}$. The patterns are measured using a circularly polarized antenna as a receiving antenna. It is seen that the radiation pattern remains nearly constant regardless of the mechanical rotation, and is almost symmetrical with respect to the helical axis. Hence, we assume a dotted line as a radiation pattern of the single array element to calculate the radiation pattern of the array by a pattern multiplication method (PMM).

We show an example of array design in the case of the power efficiency $\eta_{0}$ of 80 percent and Chebyschev distribution of the sidelobe level of $-20 \mathrm{~dB}$ in isotropic sources. When the number of the array elements is five, the distance between the array elements becomes $0.78 \lambda$.

First, the fifth array element is inserted to the waveguide and is tuned by a stub. A standing wave ratio meter is used to check the tuning condition. Subsequently, the insertion and tuning are applied one by one to the fourth, third, second, and first array elements.

The radiation pattern of five array elements without mechanical rotation and that with mechanical rotation are shown in Figs. 5(a) and 5(b), respectively. As expected, a main beam is 


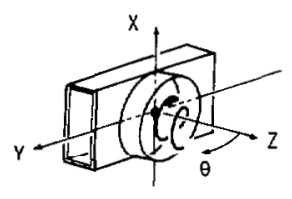

$-\phi_{\text {mech }}=0^{\circ}$

- $\phi_{\text {mech }}=45^{\circ}$

$--\infty \phi_{\text {mech }}=90^{\circ}$

$\cdots-\phi_{\text {mech }}=135^{\circ}$

$\phi=90^{\circ}$ plane
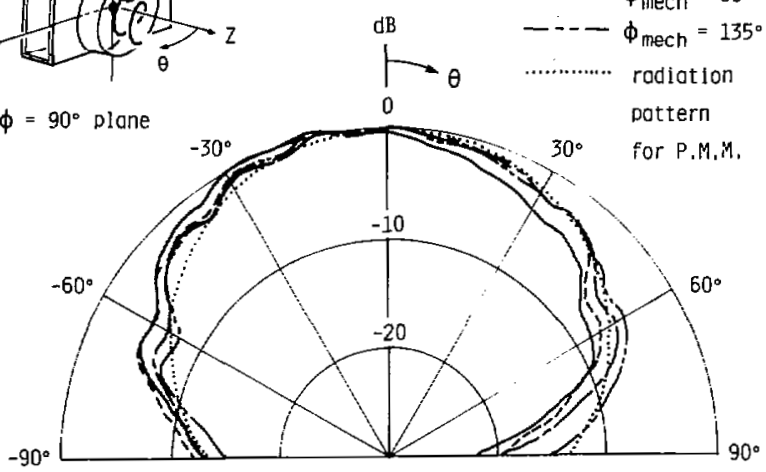

Fig. 4. Experimental radiation patterns of the single array element with the mechanical rotation of $\phi_{\text {mech. }}$.

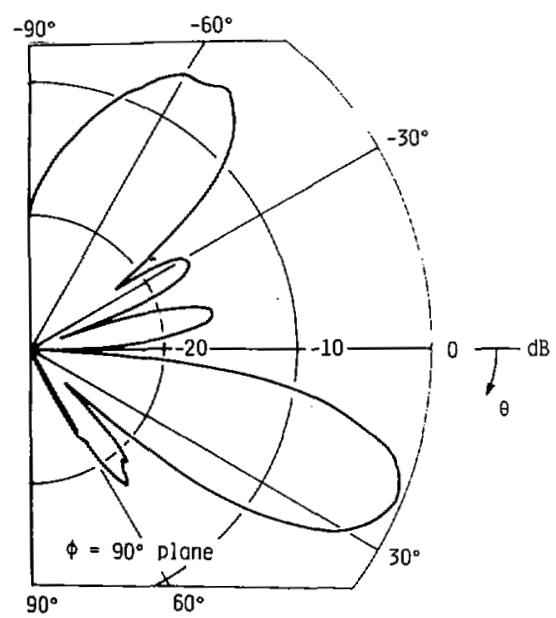

(a)

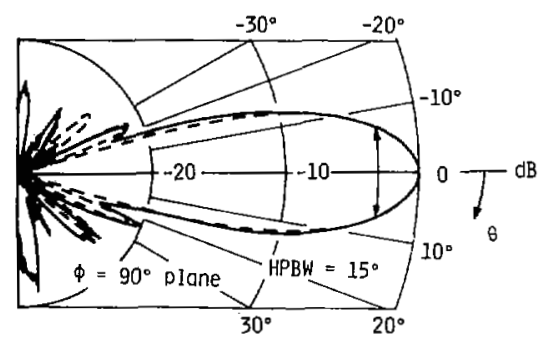

(b)

Fig. 5. Radiation patterns of the array. (a) Without mechanical rotation. (b) With mechanical rotation. The number of helical antennas is five. --- theoretical; _- experimental.

formed by the mechanical rotation of the array elements. The half-power beamwidth (HPBW) in the $\phi=90^{\circ}$ plane is $15^{\circ}$ (calculated value $15^{\circ}$ ), and the sidelobe level is $-20 \mathrm{~dB}$ (calculated value $-21 \mathrm{~dB}$ ). The axial ratio is $1.7 \mathrm{~dB}$, corresponding to the cross polarization of $-20 \mathrm{~dB}$.

When the number of the array elements is seven, the distance between the array elements becomes $0.85 \lambda$. For nine elements the distance between the array elements becomes $0.88 \lambda$. Figs. 6 (a) and $6(\mathrm{~b})$ show the radiation patterns of the arrays using seven and nine elements, respectively. The HPBW in $\phi=90^{\circ}$ plane is related to the change in the number of the array elements. The experimental HPBW's are $9^{\circ}$ and $7^{\circ}$ for seven and

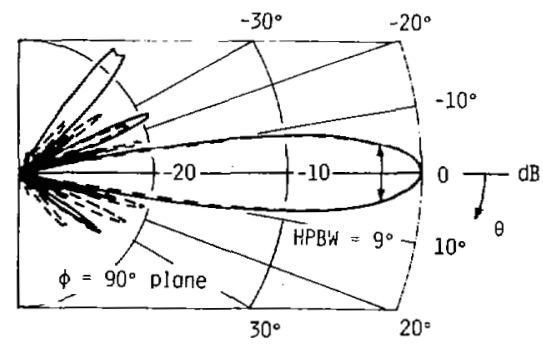

(a)

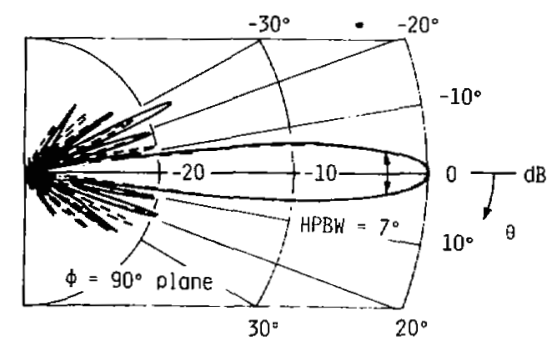

(b)

Fig. 6. Radiation patterns of the array. (a) Seven helical antennas. (b) Nine helical antennas. - - - theoretical; - experimental.

nine elements, respectively. They are in good agreement with the theoretical values.

\section{CONCLUSION}

An array of short helical antennas fed from a waveguide has been demonstrated with the object of simplifying the feeding system. As the number of array elements is decreased, the array design becomes difficult due to the reflected wave caused by the longer insertion of linear section in to the waveguide. To eliminate the reflected wave and simplify the design of the array, tuning stubs are introduced near the array elements.

The radiation power distribution is controlled by the insertion length of linear section. The coupling phase is derived by a transmission line theory. Instead of using phase shifters, the mechanical rotation is applied to each array element so that in-phase condition at the aperture may be formed.

The radiation pattern of the array is calculated by a pattern multiplication method. Excellent agreement between the calculated and experimental results is demonstrated in the arrays of five, seven, and nine helical antennas.

\section{REFERENCES}

[1] J. D. Kraus, "Helical beam antenna," Electron., vol. 20, pp. 109-111, Apr. 1974

[2] —-, Antennas. New York: McGraw-Hill, 1950, ch. 7

[3] E. T. Kornhauser, "Radiation field of helical antennas with sinusoidal current," J. Appl. Phys., vol. 22, no. 7, pp. 887-891, July 1951.

[4] T. S. Maclean and R. G. Kouyoumjian, "The bandwidth of helical antennas," IRE Trans. Antennas Propagat., vol. AP-7, special supplement, pp. S379-S386, Dec. 1959.

[5] J. L. Wong and H. E. King, "Broadband quasi-taper helical antennas," IEEE Trans. Antennas Propagat., vol. AP-27, no. 1, pp. 72-78, Jan. 1979.

[6] K. F. Lee, P. F. Wong and K. F. Larm, "Theory of the frequency responses of uniform and quasi-taper helical antenna," IEEE Trans. Antennas Propagat., vol. AP-30, no. 5, pp. 1017-1021, Sept. 1982.

[7] H. Nakano and J. Yamauchi, "Characteristics of modified spiral and helical antennas," Proc. Inst. Elec. Eng., Pt. H, vol. 129, no. 5, pp. 232-237, 1982.

[8] H. Nakano, J. Yamauchi, H. Mimaki, and M. Sugano, "The balanced 
helical antenna radiating right- and left-hand circularly polarizedwaves," Trans. IECE, vol. J63-B, no. 8, pp. 743-750, 1980.

[9] J. Yamauchi and $H$. Nakano, "Axial ratio of balanced helical antenna and ellipticity measurement of incident wave," Electron. Lett., vol. 17, no. 11, pp. $365-366,1981$.

[10] H. Nakano and M. Miyabayashi, "Array of helices coupled into a waveguide," Trans. IECE, vol. J60-B, no. 5, pp. 359-361, 1977.

[11] H. Nakano, N. Asaka, and J. Yamauchi, "Short helical antenna array fed from a waveguide," in 1983 IEEE Int. Antennas Propagat. Soc. Symp. Digest, pp. 405-408.

[12] M. J. Ehrlich, "Slot-antenna array," in Antenna Engineering Handbook, H. Jasik, Ed. New York: McGraw-Hill, 1961, ch. 9.

[13] J. D. Kraus, "Helical beam antenna for wide-band applications," Proc. IRE, vol. 36, pp. 1236-1242, Oct. 1948.

[14] H. Nakano, N. Asaka and J. Yamauchi, "Radiation characteristics of short helical antenna and its mutual coupling," Electron. Lett., vol. 20, no. 5, pp. 202-204, 1984.

[15] V. D. Agrawal and G. G. Wong, "A high performance helical element for multiple access array on TDRSS spacecraft," in 1979 IEEE Int. Antennas Propagat. Soc. Symp. Digest, pp. 481-484.

[16] T. Shiokawa and Y. Karasawa, "Array antenna composed of 4 short axial-mode helical antennas," Trans. IECE, vol. J65-B, no. 10, pp. $1267-1274,1982$.

[17] R. Rubin, "Antenna measurements," in Antenna Engineering Handbook, H. Jasik, Ed. New York: McGraw-Hill, 1961, ch. 34.

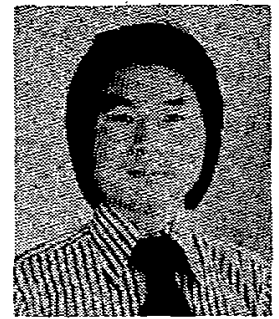

Hisamatsu Nakano (M'75) was born in Ibaraki, Japan, on April 13, 1945. He received the B.E. M.E., and Dr.E. degrees in electrical engineering from Hosei University, Tokyo, in 1968, 1970, and 1974 , respectively.

Since 1973, he has been on the Faculty of Hosei University, where he is a Professor of Electrical Engineering. In 1981 he was Visiting Associate Professor at Syracuse University, New York. He has engaged in research and development of microwave antennas. His primary interests are in thin wire antennas and scattering problems.
Dr. Nakano is a member of the Institute of Electronics and Communication Engineers of Japan.

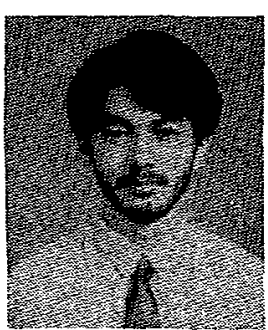

Nobuyoshi Asaka was born in Aomori, Japan, on February 25, 1959. He recieved the B.E. and M.E. degrees in electrical engineering from Hosei University, in 1982 and 1984, respectively. He engaged in the design of the helical antennas.

He joined the Matsushita Electric Industrial Co. Ltd., Osaka, in 1984.

Mr. Asaka is a member of the Institute of Electronics and Communication Engineers of Japan.

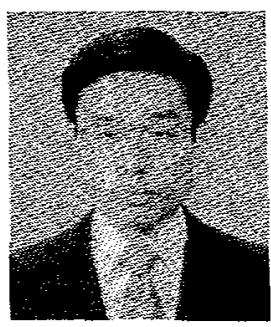

Junji Yamauchi was born in Nagoya, Japan, on August 23, 1953. He received the B.E., M.E., and Dr.E. degrees from Hosei University, Tokyo, in 1976, 1978 and 1982, respectively.

$\mathrm{He}$ is currently a Lecturer at the Electrical Engineering Department of Tokyo Metropolitan Technical College. His research interests are in thin wire antennas and application of a circularly polarized wave.

Dr. Yamauchi is a member of the Institute of Electronics and Communication Engineers of Japan. 